\title{
The Rehabilitation of Permanent Teeth with Complicated Crown Fracture by MTA Pulpotomy and Re-Attachment: Case Series Report Study and Literature Review
}

\author{
Mehmet Sinan Doğan ${ }^{1}$, Anton Rahardjo ${ }^{2}$, Yuniardini Septorini Wimardhani ${ }^{2}$ and Izzet Yavuz $^{3 *}$ \\ ${ }^{1}$ Department of Pediatric Dentistry, Faculty of Dentistry, Harran University, Şanliurfa, Turkey \\ ${ }^{2}$ Oral Epidemiology and Clinical Studies in Dentistry, Faculty of Dentistry, Universitas Indonesia, Turkey \\ ${ }^{3}$ Department of Pediatric Dentistry, Faculty of Dentistry, Dicle University, Diyarbakir, Turkey
}

Received: 23, January, 2017; Accepted: 31, January, 2017; Published: 10, February, 2017

*Corresponding author: Izzet Yavuz, Department of Pediatric Dentistry, Faculty of Dentistry, Dicle University, Diyarbakir, Turkey, E-mail: izzetyavuz@hotmail.com

\begin{abstract}
Following dental trauma, dental injuries may be seen from cracked enamel to avulsion in the teeth. In this clinical study, are presented restoration of fractured dental fragments after the proper treatment of 5 pediatric patients aged $8,9,11,11$, and 11 years who occurred complicated crown fracture and exposure to dental trauma in the anterior group of teeth between 2009 and 2013. In the same session complicated crown fracture in the anterior group of teeth were amputated with mineral trioxide agent (MTA) and it was closed with glass ionomer cement. The restoration was completed by attaching the fractured dental fragment with composite resin. This emergency treatment has special importance. Because complicated crown fractures occurring after dental trauma to the anterior group of teeth can lead to pain and complications of functions, aesthetics and psychological problems.
\end{abstract}

Keywords: Dental trauma; Complicated crown fracture; MTA

\section{Introduction}

Although oral region only constitutes $1 \%$ of the body, it is most effect of the body after trauma [1]. Epidemiological studies have reported that pre-school children are exposed to dental trauma rate of $6.1 \%-62.1 \%$ and approximately $25 \%$ of all children below the age of 18 years are exposed to trauma in anterior teeth [2-4]. The most common causes of dental trauma have been reported to be sports injuries, accidents in the home, fall and impact [5,6]. Traumatic dental injuries often result in a treatment sequence that involves both general dentists and specialists [7]. Complicated Crown Fractures (CCF), in the classification of dental trauma; occur mostly in anterior teeth, in children. Maxillary central teeth are most exposed to dental trauma because of their position in the dental arch. Function, phonation and aesthetic problems can occur in these cases [8]. Both the children who have suffered the trauma and their families are negatively affected. Children's psychological condition is effected aesthetic and functional problems caused by dental trauma. Therefore, to overcome this problem, the choice of the most appropriate and emergency treatment is important. Crown fractures have been reported to constitute $26 \%-76 \%$ of the dental trauma seen in permanent dentition and $0.3 \%-5 \%$ crown-root fractures [9]. Complicated crown fractures are injuries involving the enamel, dentine and pulp. CCF's treatment must be applied urgently to protect the vitality of the pulp. The most important a criterion of recovery is protective to the pulp against infection. Because appropriate treatment is to decide, the details of clinical and radiological examinations are important; severity of pulpal, time elapsed after the trauma, the development stage of the tooth [10].

In these types of injuries, every attempt should be used to achieve pulpal survival and healing. The direct capping is recommended treatment, if the trauma time is limited to 1 or 2 hours and in minor pulp perforation $[3,8]$. However, if the perforation area is large, or more than 24 hours has elapsed since the trauma, amputation should be preferred as the treatment.10 The American Academy of Pediatric Dentistry recommends the use of calcium hydroxide and mineral trioxide agent for these treatments. Zinc oxide eugenol, corticosteroid, calcium hydroxide, hydrophilic resins, resin-modified glass ionomer cements, tricalcium phosphate cement and mineral trioxide agent (MTA) can be used in pulp therapy of permanent dentition [11].

These fractures must be treated immediately because they not only harm the teeth but also form psychological effects on the patient and their family. Restoration can be made with various techniques and methods such as porcelain jacket crowns, ceramic restorations, and stainless steel crowns or pinned resin restorations $[8,12]$.

Despite great developments in restorative materials in recent years, restorative materials have not been produced which would meet the aesthetic and functional properties of the natural tooth structure. Re-attachment is an economic and conservative 
means of restoration of fractured teeth. The fractured fragment and the tooth are joined with the direct application of restorative materials or with support from a post system. Sticking the tooth fragment back into its own place can be achieved in a much shorter time than an extensive restoration [13]. In this way, by providing the natural shape, contour, translucency, surface tissue, occlusal conformity and colour compatibility of the fractured tooth, the patient's emotional and social status is positively affected [14]. In our study, four cases are presented that amputation treatment with MTA was applied and the fractured fragments of upper incisor teeth were attached directly with adhesive materials.

Case 1: A 9-year old male experienced dental trauma while playing a game at school. Tooth no 11 was fractured. The patient presented at our clinic 1 hour after the trauma, together with the broken tooth fragment. The fractured tooth fragment was protected within the post. In the clinical and radiological examination, a complicated crown fracture was determined and the pulp had opened. In the same session the no 11 tooth was amputated with mineral trioxide agent (MTA) and it was closed with glass ionomer cement. The restoration was completed by attaching the fractured tooth fragment with composite resin (Figures 1-3).

Case 2: An 11-year old boy fractured tooth no 11 in a fall while playing a game. The patient presented at our clinic 1 hour after the trauma together with the fractured tooth fragment. In the clinical and radiological examinations a complicated crown fracture was determined in tooth no 11 . The patient had kept the fractured tooth fragment dry. The pulp of tooth no 11 was removed as far as the coronal third and was closed with MTA. Glass ionomer cement closure was applied over the MTA and the fractured tooth fragment was attached with composite resin. At 1-month, polishing was performed to the anterior teeth of patient. 6-month follow-up examinations, no symptoms were observed (Figures 4-6).

Case 3: A 10-year old girl presented at our clinic with a fracture in tooth no 21 following dental trauma. In the clinical and radiological examinations a complicated crown fracture was determined in tooth no 21 . It was noticed that there was

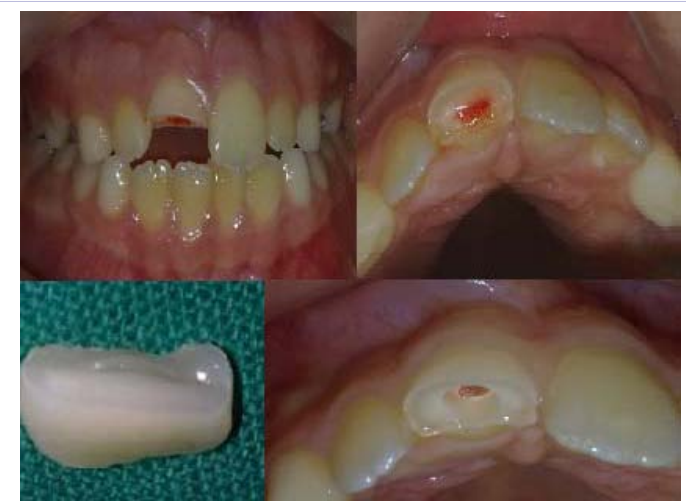

Figure 1: Case 1 intraoral view and fracture tooth fragment before the treatment.

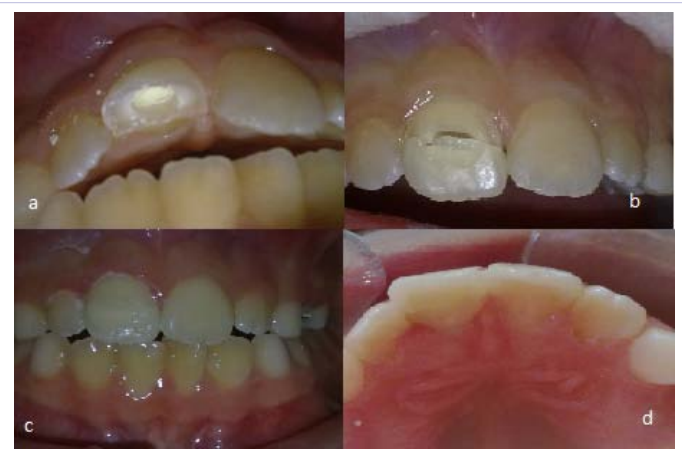

Figure 2a,b,c,d: Case 1 intraoral view. Pulpatomy with MTA (a). Fracture tooth fragment repozisyon (b). The vestibule view after the treatment (c). The palatinal view after the treatment (d).

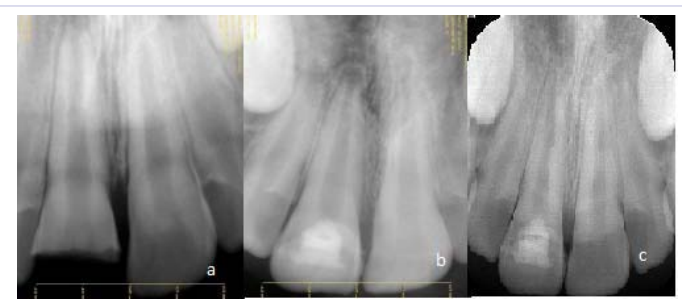

Figure 3a,b,c: Case 1 the radiographic view. Before treatment (a). The radiographic view after 2 month (b). After six month (c).

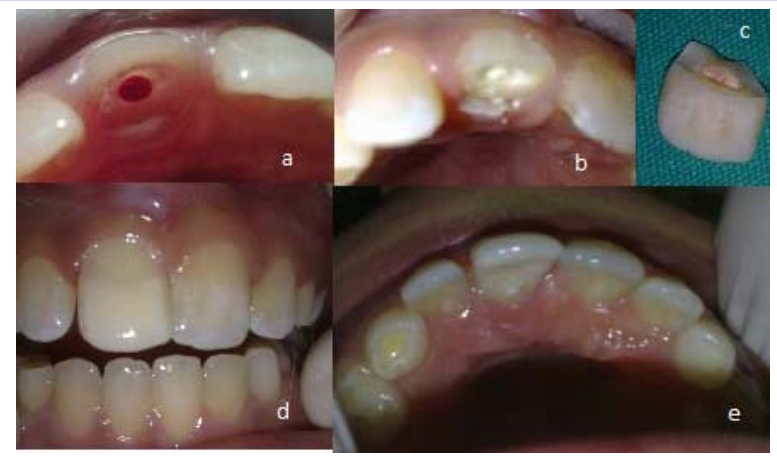

Figure 4a,b,c,d,e: Case 2 intraoral view. Pulpatomy with MTA (a,b). Fracture tooth fragment (c). The vestibule view after the treatment (d). The palatinal view after the treatment (e).

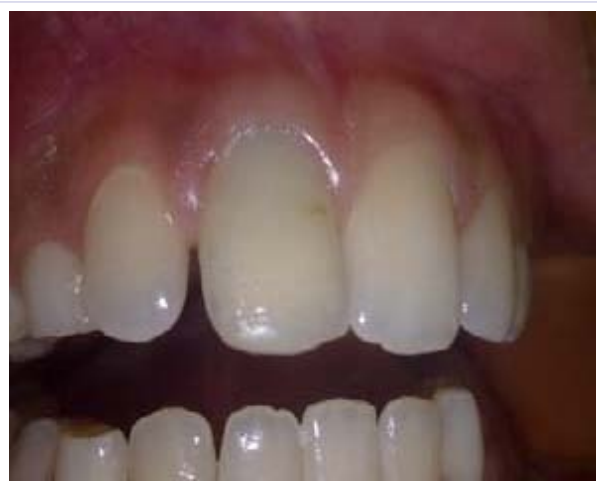

Figure 5: Case 2 intraoral view after six month. 
only a soft tissue connection of the fractured tooth fragment. After amputation of the tooth with MTA, the fractured fragments were attached with composite resin. Periodontal treatment was applied to the patient. The patient was followed up (Figures 7,8).

Case 4: An 11-year old boy presented at our clinic with a partial fracture of tooth no 11 as a result of a collision while playing a game. In the clinical and radiological examinations a complicated crown fracture was determined in tooth no 11. After amputation of the tooth with MTA, the fractured fragments were attached with composite resin. At the 1-month followup examination, no symptoms were observed (Figures 9,10). Unfortunately, he was exposed to second dental trauma, and was occurred pulp necrosis. Then, tooth was performed endodontic treatment.

Case 5: An 11-year old boy experienced fractures in teeth no 11 and 12 as a result of dental trauma and from the anamnesis it was learned that an agent containing calcium hydroxide had been applied to the teeth by the dental practitioner he first consulted. The patient presented at our clinic for treatment of the teeth. In the clinical and radiological examinations, complicated crown fractures were determined in teeth no 11 and 12 (Figures 11a,b,c,13a). The patient had kept the fragments dry. After removal of the agent from the teeth, the pulp of tooth no 11 was removed as far as the coronal third and was closed with MTA. As there was extensive opening of the pulp of tooth no 12, cervical amputation was applied and was closed with MTA (Figures

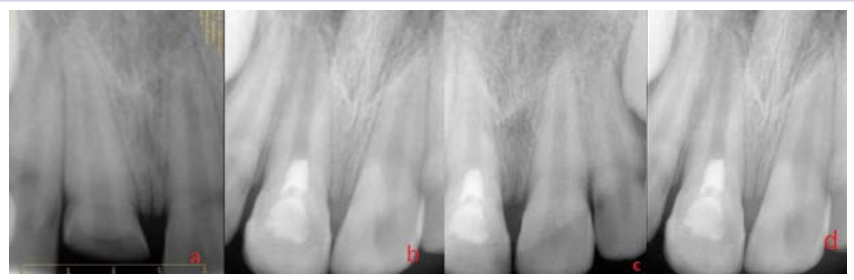

Figure 6a,b,c,d,e: Case 2 the radiographic view. Before treatment (a). The radiographic view after 6 month (b). The radiographic view after 2 years (c), 3 years $(d)$.

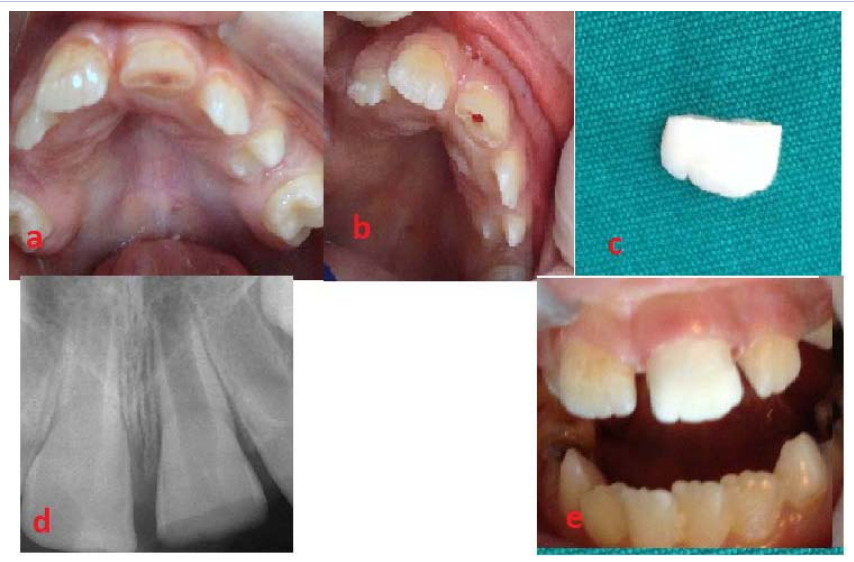

Figure 7a,b,c,: Case 3. Intraoral view before treatment (a).Pulpatomy with MTA $(b, c)$. The vestibule view after the treatment (d).

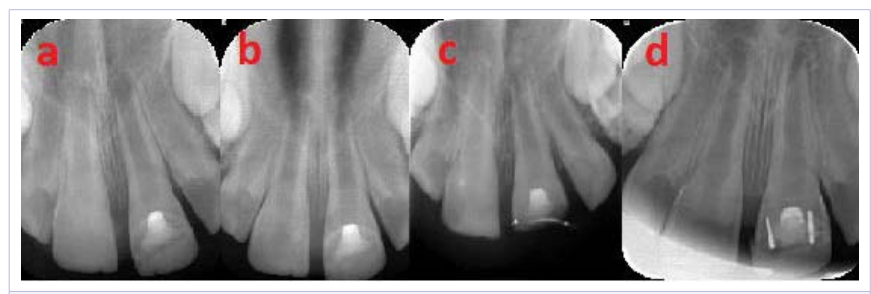

Figure 8a,b,c,: Case 3. the radiographic view after treatment. After one month (a), two month (b), six month (c), one years (d).

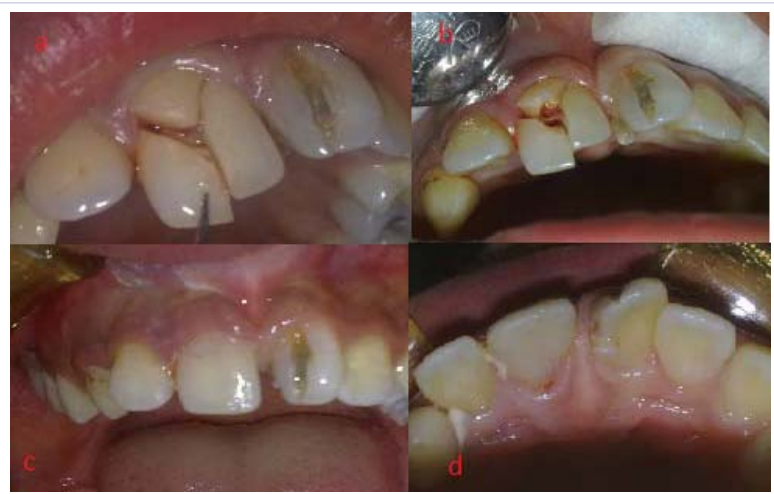

Figure 9a,b,c,: Case 4. Intraoral view before treatment (a).Pulpatomy with MTA (b).The vestibule view after the treatment (c). The palatinal view after the treatment (d).

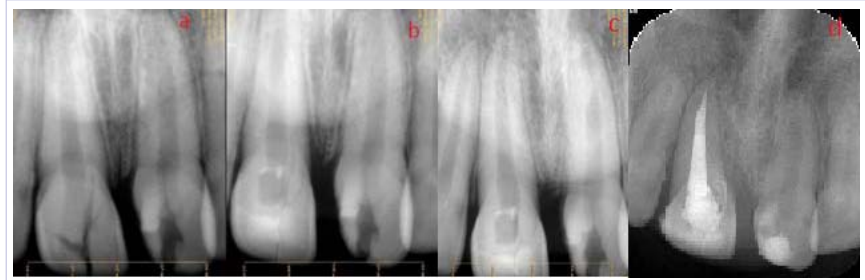

Figure 10a,b,: Case 4. the radiographic view. Before treatment (a).The radiographic view after treatment 1 month (b), second trauma (c), after second trauma five months ( $d$, after first trauma two years).

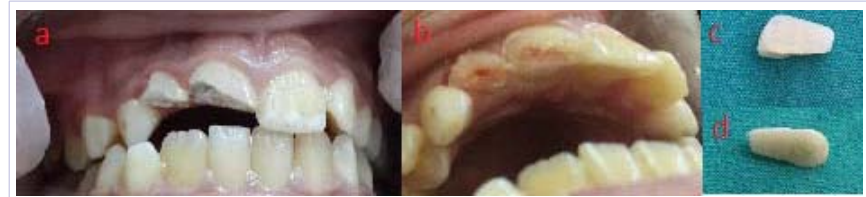

Figure 11a,b,c: Case 5. Intraoral view before treatment (a,b). Fracture santral incisor tooth fragment (c). Fracture lateral incisor tooth fragment (d).

12a,b). Glass ionomer cement closure was applied over the MTA and the fractured tooth fragment was attached with composite resin (Figure 12c). At the 1-month follow-up examination, no symptoms were observed (Figure 13b).

\section{Discussion}

Epidemiological studies have reported that approximately $50 \%$ of children aged 8-12 years' exposure to dental trauma. In 


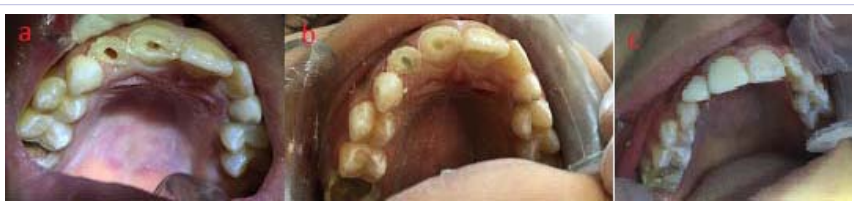

Figure 12a,b,c: Case 5. Intraoral view during treatment (a).Pulpatomy with MTA (b).The vestibule view after the treatment (c).

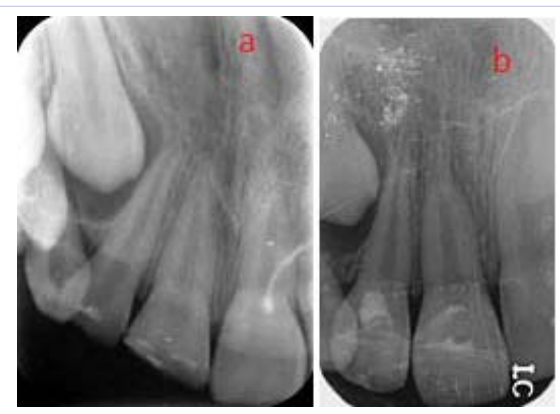

Figure 13a,b: Case 5 the radiographic view. Before treatment (a).The radiographic view after treatment 1 month (b).

the majority of dental trauma cases, rapid and effective treatment is necessary in respect of both aesthetics and oral health. Dental fractures in permanent dentition may cause psychological problems both for the child and their family. In the evaluation of patients with fractures of the anterior teeth, the clinician should provide both a functional and aesthetic perspective. Treatment options may vary depending on the socio-economic status of the patient, the intraoral status at the time of planning the treatment and the age of the patient [15]. The maxillary central teeth are most often affected teeth due to dental trauma. Loss of hard tissue formed in the tooth because of orthodontic anomalies or caries increases risk of dental trauma [16]. In our study, orthodontic anomalies (diastema and inexplicit) are presented in five cases and all cases were fractured of maxillary central teeth. Additionally, in four cases, CCF was occurred in right central incisor, while case 3 was occurred in left central incisor. In case 5 , both central incisor and lateral incisor were occurred to CCF.

As a treatment option in complicated crown fractures, partial amputation is recommended whether or not the immature root. Vital amputation is a treatment which provides the creation of a neo formative dentine layer below the cut area, by removing the part of the pulp which is thought to be infected, and when the pulp is perforation as a result of trauma or decay and the tooth remains vital $[17,18]$. There are various advantages to partial pulpotomy compared to cervical pulpotomy. The cell content of coronal pulp is greater than that of root pulp and this is required for physiological a positioning of the dentine in the coronal area and healing. In cervical pulpotomy, there is no physiological dentine apposition in the crown and there is an increased risk of cervical fracture [18]. Case 1 and case 3 were not complete the developing root, and applied to our clinic shortly after dental trauma. They were performed partial pulpotomy. Apical closure and root development were continued in fractured teeth after dental trauma.
The pulpotomy, especiallyin dental injuries, can be easily defined because the appearance of the healthy pulp can be differentiated by evaluation of the amount of bleeding and colour. The most significant advantage of partial amputation compared to capping is that the pulp can be covered with a material of a thickness to provide sufficient impermeability. Calcium hydroxide is generally preferred in the treatment of vital pulp [18]. As calcium hydroxide has a high $\mathrm{pH}$, it provides an alkaline environment, which is necessary for bone and dentine formation [19]. Calcium hydroxide ions cause coagulation necrosis below the application area, then cells in the pulp tissue are differentiated from odontoblast-like cells and predentine is synthesized. Finally, predentine becomes dentine.18In recent years, MTA, which was developed by Torabinejad et al, has often been used in cases of pulp capping and pulpotomy. The effect of MTA on pulp and periapical tissue is similar to the effect of calcium hydroxide. It has been reported that the possibility of pulp necrosis forming is lower with the use of MTA than with calcium hydroxide. That there could be bacterial invasion in the tunnel defects created under calcium hydroxide has also been reported [20]. In addition, in cases where MTA is used, the dentine bridge has been shown to be thicker and more regular, the rate of bridge formation is greater and as the impermeability is very good, the underlying tissues are better protected with less inflammation and hyperemia [20]. Following amputations made with MTA, it has been reported that colouring in the teeth may be seen [21]. Therefore, the use of white-coloured MTA is recommended [22]. For these reasons, we used to MTA for pulpotomy in our cases. However, in one cases (Case 4), pulpotomy with MTA was not successful due to pulp necrosis. In one of the current cases of complicated crown fracture (Case 1), although we used whitecoloured MTA, colouring of the tooth developed in the cervical area.

The bismuth oxide content of the MTA that reacted with the collagen in the dentinal matrix caused the discoloration [23]. Moreover; other factor that might be related to the esthetic of the treatment was the time span between trauma and treatment. It was said that the possibility of maintaining of tooth's natural color, translucency and hue was increased when there was shorter duration dehydration of the tooth fragment [24].

High success rates (94\%-96\%) have been reported in complicated crown fractures treated with partial amputation [22]. Svizero et al (5) treated complicated crown fractures with closed apex in permanent anterior incisor teeth with partial amputation and reported no pathological findings at the end of a 250-day follow-up period [25]. Parirokh and Kakoei reported that canal obliteration could be seen years after trauma but calcification formation should be seen as related to continuing pulp function and not as a finding of pulp degeneration [26]. The re-attachment of fracture fragments is less time-consuming than prosthetic or composite restorations and is known to be aesthetically advantageous. It has also been stated that as the restoration formed by re-attaching the fracture fragments has the original margin and contours, there will be fewer gum problems [27]. 
All 5 cases presented in these cases reports had a complicated crown fracture. To all these cases of complicated crown fracture, pulpotomy was applied with MTA and the restoration was completed by re-attaching the fractured fragments.

Although the re-attachment of fracture fragments is the most important option in respect of aesthetic appearance, it has been reported that the fracture fragments are more sensitive than other teeth to fracture from a second trauma. It is accepted that the reattachment of the fragment is considered a temporary procedure, the other restorative types such as direct adhesives, veneers and crowns are the options in the event of treatment failure [28]. The application of prosthesis is a suitable option but it is thought that the excessive preparation of the remaining tooth tissue could create a biological and mechanical risk. If the fractured tooth is in the active eruption phase, direct composite restoration has been recommended as the treatment [15]. Reports and clinical experience have shown successful short and mid-term results from the re-attachment technique. In long-term studies of restorations formed by re-attachment of the fractured fragments, more successful results have been reported from these teeth than from composite resin restorations. Similarly, in vivo studies have shown successful short and long-term results from the re-attachment of coronal fracture fragments [29]. It has also been shown that the better compatibility of the fractured tooth fragments has a positive effect on the tooth stability and the natural tooth surface has successful biocompatibility with the periodontium [30]. In our cases, we preferred to re-attachment of fracture fragments for treatment. In our cases, periodontal problems were not occurred at long term follow-up.

Naudi and Fung re-attached fracture fragments with composite resin in a case of fragmented crown fracture. However, the patient experienced a second trauma after 17 months and periapical pathology was determined in the tooth that had been previously damaged [31]. In a study by E. Stelline et al, greater adhesive force was reported to be achieved by preparation of the fracture line of the fractured tooth [32]. In the cases with complicated crown fracture presented in these cases reports, bizotaj was applied to the fracture line and the fracture fragments were attached with composite resin. Enamels of fractured fragments were applied bizotaj because of the teeth to be resistant. However, again, case 3 was occurred to crown fracture at end of the second dental trauma. This time, we used dentin pin for the purpose of re-attachment of fracture fragment. Case 4 was occurred pulp necrosis and root treatment.

Immediately, suitable and timely treatments to be applied to tooth injuries resulting from dental trauma have a significant effect on the prognosis of the tooth which has been exposed to trauma. Community awareness of dental injuries and the complications which could develop following injury is of great importance. These injuries which can lead to pain function, aesthetic and psychological problems require emergency intervention and a particular treatment approach to be able to achieve restoration and regain health. It has been seen that treated patients have been able to regain dental health after emergency intervention and the teeth can be used again in a healthy manner. We think that are a successful treatment with MTA pulpotomy and reattachment of patients exposure to complicated crown fracture.

\section{References}

1. Güngör HC. Management of crown-related fractures in children: an update review. Dent Traumatol. 2014;30(2):88-99. doi: 10.1111/ edt.12079

2. Choi SC, Park JH, Pae A, Kim JR. Retrospective study ontraumatic dental injuries in preschool children at Kyung Hee Dental Hospital, Seoul, South Korea. Dent Traumatol. 2010;26(1):70-75. doi: 10.1111/j.16009657.2009.00840.x

3. Rasmusson CG, Koch G. Assessment of traumatic injuries to primary teeth in general practise and specialized paediatric dentistry. Dent Traumatol. 2010;26(2):129-132. doi: 10.1111/j.16009657.2009.00862.x

4. Jesus MA, Antunes LA, Risso Pde A, Freire MV, Maia LC. Epidemiologic survey of traumatic dental injuries in childrenseen at the Federal University of Rio de Janeiro, Brazil. Braz Oral Res. 2010;24(1):89-94. DOI: $10.1590 /$ S1806-83242010000100015

5. Keels MA. Management of Dental Trauma in a Primary Care Setting. Pediatrics. 2014;133(2):466-476.

6. Chowdary GN, Hemalatha R, Vijayakumar R, Ganesh R, Selvakumar H, Mangaiyarkarasi S. Prevalence of traumatic dental injuries in primary teeth: A retrospective study. SRM J Res Dent Sci. 2014;5(1):11-13. DOI: $10.4103 / 0976-433 X .129056$

7. Andreasen JO, Lauridsen E, Gerds TA, Ahrensburg SS. Dental Trauma Guide: A source of evidence-based treatment guidelines for dental trauma. Dent Traumatol. 2012;28(2):142-147. doi: 10.1111/j.16009657.2011.01059.x

8. de Castro JC, Poi WR, Pedrini D, Tiveron AR, Brandini DA, de Castro MA. Multidisciplinary approach for the treatment of a complicated crown-root fracture in a young patient: A case report. Quintessence Int. 2011;42(9):729-735.

9. Maitin N, Maitin S, Rastogi K. Bhushan R. Aesthetic management of a complicated crown fracture: a multidisciplinary approach. BMJ Case Rep. 2013. doi: 10.1136/bcr-2013-009643

10. Asgary S, Fazlyab M. Management of Complicated Crown Fracture with Miniature Pulpotomy: A case report. Iran Endod J. 2014;9(3):233-234.

11. Parolia A, Kundabala M, Rao NN, Acharya SR, Agrawal P, Mohan M, et al. A comparative histological analysis of human pulp following direct pulp capping with Propolis, mineral trioxide aggregate and Dycal. Aust Dent J. 2010;55(1):59-64. doi: 10.1111/j.1834-7819.2009.01179.x

12. Badami AA, Dunne SM, Scheer B. An in vitro investigation into the shear bond strengths of two dentine-bonding agents used in the reattachment of incisal edge fragments. Endod Dent Traumatol. 1995;11(3):129-135.

13. Tosun G, Yildiz E, Elbay M, Sener Y. Reattachment of fractured maxillary incisors using fiber-reinforced post: Twocase reports. Eur J Dent. 2012;6(2):227-233.

14.Vishwanath B, Faizudin U, Jayadev M, Shravani S. Reattachment of coronal tooth fragment: regaining back to normal. Case Rep Dent. 2013;2013(2013):1-5.

15. Rajavardhan K, A.J. Sai Sankar, Tanveer Ahmed Shaik, Naveen Kumar V, Raj Kumar K . A Novel Technique in Restoring Fractured 
Anterior Teeth. J Clin Diagn Res. 2014;8(2):244-245. doi: 10.7860/ JCDR/2014/7464.4071

16. Cheng LL, Moor SL, Ho CT. Predisposing factors to dental caries in children with cleft lip and palate: a review and strategies for early prevention. Cleft Palate Craniofac J. 2007;44(1):67-72.

17. Preben H, Lovschall H. Treatment outcome of vital pulp treatment. Endodontic Topics. 2002;2(1):24-34. doi: 10.1034/j.16011546.2002.20103.x

18. Chailertvanitkul P, Paphangkorakit J, Sooksantisakoonchai N, Pumas $\mathrm{N}$, Pairojamornyoot W, Leela-Apiradee N, et al. Randomized control trial comparing calcium hydroxide and mineral trioxide aggregate for partial pulpotomies in cariously exposed pulps of permanent molars. Int Endod J. 2014;47(9):835-842. doi: 10.1111/iej.12225

19. Mohammadi Z, Dummer PM. Properties and applications of calcium hydroxide in endodontics and dental traumatology. Int Endod J. 2011;44(8):697-730. doi: 10.1111/j.1365-2591.2011.01886.x

20.Witherspoon DE, Small JC, Harris GZ. Mineral trioxide aggregate pulpotomies. J Am Dent Assoc. 2006;137(5):610-618.

21. Ward J. Vital pulp therapy in cariously exposed permanent teeth and its limitations. Aust Endod J. 2002;28(1):29-37.

22. Karabucak B, Li D, Lim J, Iqbal M. Vital pulp therapy with mineral trioxide aggregate. Dent Traumatol. 2005;21(4):240-243. doi: 10.1111/j.1600-9657.2005.00306.x

23. Marciano MA, Costa RM, Camilleri J, Mondelli RF, Guimarães BM Duarte MA. Assessment of color stability of white mineral trioxide aggregate angelus and bismuth oxide in contact with tooth structure. J Endod. 2014; 40(8):1235-1240. doi: 10.1016/j.joen.2014.01.044

24. Ramírez-Sebastià A, Bortolotto T, Cattani-Lorente M, Giner L, Roig M, Krejci I. Adhesive restoration of anterior endodontically treated teeth: influence of post length on fracture strength. Clin Oral Investig. 2014;18(2):545-554. doi: 10.1007/s00784-013-0978-3

25. SvizeroNda R, Bresciani E, Francischone CE, Franco EB, Pereira JC. Partial pulpotomy and tooth reconstruction of a crown-fractured permanent incisor: a case report. Quintessence Int. 2003;34(10):740747.

26. Parirokh M, Kakoei S. Vital pulp therapy of mandibular incisors: a case report with 11year follow up. Aust Endod J. 2006;32(2):75-78. doi: 10.1111/j.1747-4477.2006.00021.x

27. Arora R, Shivakumar B, Rao HM, Vijay R. Rehabilitation of complicated crown-root fracture by fragment reattachment and intraradicular splinting: case reports. J Int Oral Health. 2013;5(5):129-138.

28. Cagidiaco MC, Goracci C, Garcia-Godoy F, Ferrari M. Clinical studies of fiber posts: a literature review. Int J Prosthodont. 2008;21(4):328336.

29. Dogan MC, Akgun EO, Yoldas HO. Adhesive tooth fragment reattachment with intentional replantation: 36-month followup. Dent Traumatol. 2013;29(3):238-242. doi: 10.1111/j.16009657.2012.01144.x

30.Sargod SS, Bhat SS. A 9 year follow-up of a fractured tooth fragment reattachment. Contemp Clin Dent. 2010;1(4): 243-245. doi: 10.4103/0976-237X.76392

31. Naudi AB, Fung DE. Tooth fragment reattachment in multiple complicated permanent incisor crown root fractures-a report of two cases. Dent Traumatol. 2008;24(2):248-252. doi: 10.1111/j.16009657.2007.00526.x

32. Stellini E, Stomaci D, Stomaci M, Petrone N, Favero L. Fracture strength of tooth fragment reattachments with postpone bevel and over contour reconstruction. Dent Traumatol. 2008;24(3):283-288. doi: 10.1111/j.1600-9657.2007.00539.x 